\title{
Should people with psychosis be supported in choosing cognitive therapy as an alternative to antipsychotic medication: A commentary on a commentary
}

\author{
Paul Hutton ${ }^{1 *}$
}

\author{
${ }^{1}$ School of Health and Social Care \\ Edinburgh Napier University \\ *Corresponding author p.hutton@ napier.ac.uk
}

Having collaborated with Anthony Morrison for a number of years, there is no doubt in my mind that his primary objective is to improve the acceptability, effectiveness and safety of interventions for people with psychosis. In his article (A. P. Morrison, 2018) he is correct to say the effectiveness of antipsychotics have been overestimated and the adverse effects underestimated (Hutton et al., 2012; Hutton, Taylor, Mulligan, Tully, \& Moncrieff, 2015; Leucht et al., 2017; Anthony P. Morrison, Hutton, Shiers, \& Turkington, 2012), and he should be commended for producing world-leading evidence on the effectiveness and safety of alternative approaches, such as cognitive behavioural therapy (CBT) (Anthony P Morrison et al., 2014). We also agree that people with psychosis need much more choice regarding the interventions offered to them (Diana Stovell, Wearden, Morrison, \& Hutton, 2016), That being said, a number of arguments he raised in his commentary do not withstand scrutiny.

First, he downplays the possibility that psychological interventions such as CBT cause harm. As he and I have discussed on several occasions, the measurment and reporting of adverse effects in trials of psychological interventions for psychosis (and other conditions) is extremely poor. Although he agrees we need to change this, as acknowledged in his article and as reflected in the adverse effect monitoring protocol that we developed for his recent trial (Pyle et al., 2016), one obstacle to doing so is complacency on the part of leaders in the field. Trials are rarely powered to detect adverse effects, meaning the absence of significant effects in the selected trials that have measured this does not prove evidence of absence. Thus, it is imperative that we do not jump to conclusions or offer false reassurance.

Second, and perhaps more importantly, his statement that many inpatients with psychosis have capacity to make decisions about their treatment both minimises and sidesteps a number of serious issues. We know that between $50 \%$ and $80 \%$ of inpatients with psychosis are judged to lack treatment decision-making capacity (Okai et al., 2007) and we know that this is likely to lead to involuntary and coercive treatment (Larkin \& Hutton, 2017). Denying this clinical reality will simply result in an impasse between those who work with acutely unwell patients with psychosis and those who wish to develop more benign treatments, but the only people who will suffer from this are people with psychosis. Although he notes our metaanalysis of shared treatment decision-making found this approach can improve subjective empowerment (D. Stovell, Morrison, Panayiotou, \& Hutton, 2016), he does not mention our finding that it does not seem to lead to improved capacity.

We have argued elsewhere that researchers and clinicians should invest significant time and effort into developing effective ways to help people with psychosis regain and retain treatment decision-making capacity (Larkin \& Hutton, 2017). Ideally, this would involve working collaboratively with people with psychosis and their clinicians to identify and reduce internal threats to capacity, such as symptoms and cognitive impairmemt, but also external 
threats, such as coercion and provision of partial information (Burns et al., 2011; Larkin \& Hutton, 2017). Failure to develop this evidence will simply lead to people with psychosis not receiving effective decision-making support.

If we wish to truly improve outcomes for people with psychosis, we need to dedicate ourselves to researching the benefits and costs of our interventions and we need to do all we can to support them to make their own decisions. This requires full committment by leaders in the field, and nothing less.

\section{Declaration of interest}

There was no funding for this work. The author has been a co-investigator on NIHR-funded trials of psychological therapy for people with psychosis, and is a member of the National Institute for Health and Care Excellence Committee which is developing new guidance on supporting decision-making and mental capacity (Decision-Making and Mental Capacity; GID-NG10009).

\section{References}

Burns, T., Yeeles, K., Molodynski, A., Nightingale, H., Vazquez-Montes, M., Sheehan, K., \& Linsell, L. (2011). Pressures to adhere to treatment ('leverage') in English mental healthcare. British Journal of Psychiatry, 199(2), 145-150. https://doi.org/10.1192/bjp.bp.110.086827

Hutton, P., Morrison, A. P., Yung, A. R., Taylor, P. J., French, P., \& Dunn, G. (2012). Effects of drop-out on efficacy estimates in five Cochrane reviews of popular antipsychotics for schizophrenia. Acta Psychiatrica Scandinavica, 126(1). https://doi.org/10.1111/j.1600-0447.2012.01858.x

Hutton, P., Taylor, P. J., Mulligan, L., Tully, S., \& Moncrieff, J. (2015). Quetiapine immediate release v. placebo for schizophrenia: systematic review, meta-analysis and reappraisal. The British Journal of Psychiatry: The Journal of Mental Science, 206(5). https://doi.org/10.1192/bjp.bp.114.154377

Larkin, A., \& Hutton, P. (2017). A systematic review and meta-analysis of factors that help or hinder treatment decision-making capacity in psychosis. British Journal of Psychiatry.

Leucht, S., Leucht, C., Huhn, M., Chaimani, A., Mavridis, D., Helfer, B., ... Davis, J. M. (2017). Sixty years of placebo-controlled antipsychotic drug trials in acute schizophrenia: Systematic review, Bayesian meta-analysis, and meta-regression of efficacy predictors. American Journal of Psychiatry. https://doi.org/10.1176/appi.ajp.2017.16121358

Morrison, A. P. (2018). Should people with psychosis be supported in choosing cognitive therapy as an alternative to antipsychotic medication: A commentary on current evidence. Schizophrenia Research.

Morrison, A. P., Hutton, P., Shiers, D., \& Turkington, D. (2012). Antipsychotics: Is it time to introduce patient choice? British Journal of Psychiatry. https://doi.org/10.1192/bjp.bp.112.112110

Morrison, A. P., Turkington, D., Pyle, M., Spencer, H., Brabban, A., Dunn, G., ... Hutton, P. (2014). Cognitive therapy for people with schizophrenia spectrum disorders not taking antipsychotic drugs: a single-blind randomised controlled trial. Lancet, 383(9926), 1395-403. https://doi.org/10.1016/S0140-6736(13)62246-1 
Okai, D., Owen, G., Mcguire, H., Singh, S., Churchill, R., \& Hotopf, M. (2007). Mental capacity in psychiatric patients : Systematic review. The British Journal of Psychiatry, 191(4), 291-297. https://doi.org/10.1192/bjp.bp.106.035162

Pyle, M., Norrie, J., Schwannauer, M., Kingdon, D., Gumley, A., Turkington, D., ... Morrison, A. P. (2016). Design and protocol for the Focusing on Clozapine Unresponsive Symptoms (FOCUS) trial: A randomised controlled trial. BMC Psychiatry, 16(1). https://doi.org/10.1186/s12888-016-0983-6

Stovell, D., Morrison, A. P., Panayiotou, M., \& Hutton, P. (2016). Shared treatment decisionmaking and empowerment-related outcomes in psychosis: Systematic review and metaanalysis. British Journal of Psychiatry, 209(1). https://doi.org/10.1192/bjp.bp.114.158931

Stovell, D., Wearden, A., Morrison, A. P., \& Hutton, P. (2016). Service users' experiences of the treatment decision-making process in psychosis: A phenomenological analysis. Psychosis: Psychological, Social and Integrative Approaches. https://doi.org/10.1080/17522439.2016.1145730 